\title{
Cutaneous Complications Associated With Intraosseous Access Placement
}

Emily Konopka, MD; Kirsten Webb, MD; Jeave Reserva, MD; Lauren Moy, MD; Hieu Ton-That, MD; Jodi Speiser, MD; Rebecca Tung, MD

\section{PRACTICE POINTS}

- Intraosseous (IO) access provides rapid vascular access for the delivery of fluids, drugs, and blood products in emergent situations.

- Bullae are potential complications from IO line placement.
Intraosseous (IO) access provides a potentially lifesaving means of vascular access in settings of trauma and advanced cardiovascular life support in which patients often require prompt and large volumes of fluid resuscitation, blood products, and medications. An additional benefit of 10 access is the rare incidence of complications, with many studies reporting rates of less than $1 \%$. The most commonly cited complications include compartment syndrome, osteomyelitis, traumatic bone fracture, and epiphyseal plate damage. To evaluate the dermatologic sequelae, we performed a retrospective chart review spanning 18 consecutive months to identify patients who underwent $I O$ line placement, either at or en route to a large metropolitan level I trauma center in the Midwestern United States. Our review identified a complication rate of $2.7 \%$, with complications including compartment syndrome, needle breakage, and a previously unreported cutaneous complication of traumatic bullae.

Cutis. 2021;107:E31-E33.

o ntraosseous (IO) access can afford a lifesaving means of vascular access in emergency settings, as it allows for the administration of large volumes of fluids, blood products, and medications at high flow rates directly into the highly vascularized osseous medullary cavity. ${ }^{1}$ Fortunately, the complication rate with this resuscitative effort is low, with many reports demonstrating complication rates of less than $1 \% .^{2}$ The most commonly reported complications include fluid extravasation, osteomyelitis, traumatic bone fracture, and epiphyseal plate damage. ${ }^{1-3}$ Although compartment syndrome and skin necrosis have been reported, ${ }^{4,5}$ there is no comprehensive list of sequelae resulting from fluid extravasation in the literature, and there are no known studies examining the incidence and types of cutaneous complications. In this study, we sought to evaluate the dermatologic impacts of this procedure.

\section{Methods}

We performed a retrospective chart review approved by the institutional review board at a large metropolitan level I trauma center in the Midwestern United States spanning 18 consecutive months to identify all patients who underwent IO line placement, either en route to or upon arrival at the trauma center. The electronic medical records of 113 patients (age range, 10 days-94 years) were identified using either an automated natural language look-up program with keywords including intraosseous access and $I O$ or a Current Procedural Terminology code 36680. Data including patient age, reason for IO insertion, anatomic location of the $\mathrm{IO}$, and complications secondary to IO line placement were recorded.

\section{Results}

We identified an overall complication rate of $2.7 \%$ (3/113), with only 1 patient showing isolated cutaneous

From Loyola University Chicago, Illinois. Dr. Konopka is from the Stritch School of Medicine. Drs. Webb, Reserva, Moy, Speiser, and Tung are from the Division of Dermatology. Dr. Speiser also is from the Department of Pathology. Dr. Ton-That is from the Department of General Surgery, Section of Trauma Surgery and Section of Surgical Critical Care.

The authors report no conflict of interest.

Correspondence: Emily Konopka, MD, 751 N Rutledge St, Springfield, IL 62702 (ekonopka72@siumed.edu).

doi:10.12788/cutis.0303 
complications from IO line placement. The complications in the first 2 patients included compartment syndrome following IO line placement in the right tibia and needle breakage during IO line placement. The third patient, a 30-year-old heart transplant recipient, developed tense bullae on the left leg 5 days after a resuscitative effort required $\mathrm{IO}$ access through the bilateral tibiae. The patient had received vasopressors as well as $750 \mathrm{~mL}$ of normal saline through these access points. Two days after resuscitation, she developed an enlarging ecchymosis around the left IO access point. On day 5, cutaneous findings included 2 large firm bullae on a purpuric base overlying the left proximal tibia and patella (Figure 1). After an ultrasound revealed no connection to the underlying joint space, the bullae were incised and drained for patient comfort, as she reported pain and undue pressure at the knee joint on initial dermatologic consultation. These symptoms abated after the procedure. Cultures of bullous fluid were negative for infection. Histopathologic examination revealed a subepidermal split with underlying minimal mixed inflammation, favoring the diagnosis of traumatic bullae.

At a scheduled 7-month dermatology follow-up, the wound bed appeared to be healing well with surrounding scarring with no residual bleeding or drainage (Figure 2) despite the patient reporting a protracted course of wound healing requiring debridement due to eschar formation and multiple follow-up appointments with the wound care service.

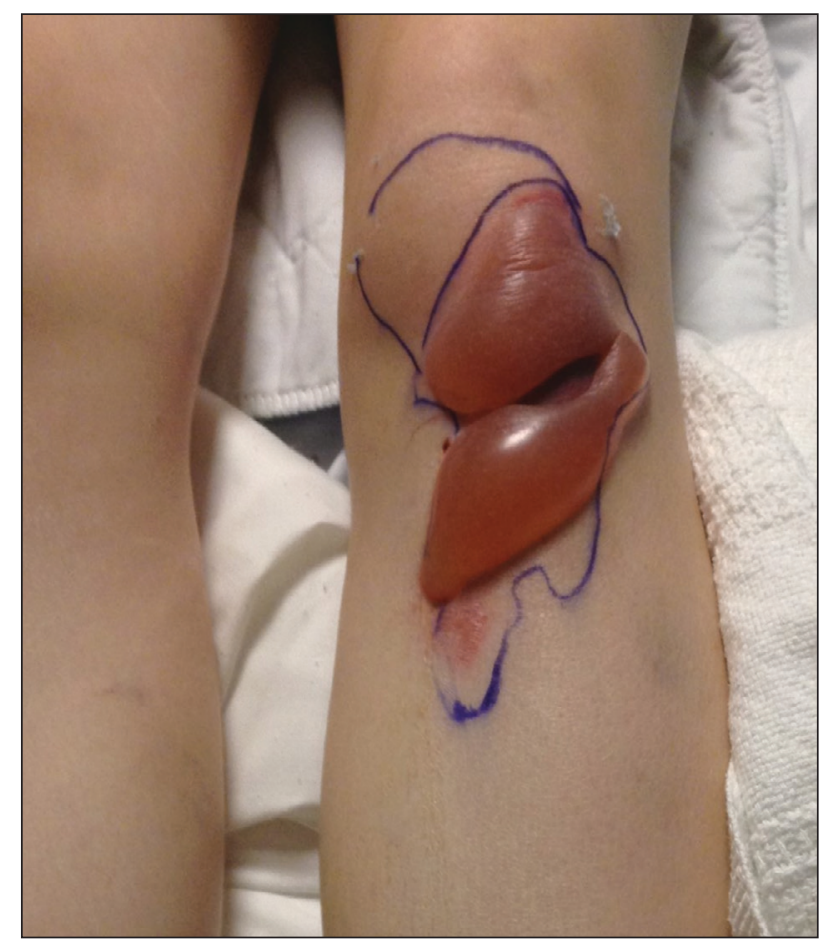

FIGURE 1. Two large, tense, fluid-filled bullae at the site of intraosseous access overlying the left proximal tibia and patella.

\section{Comment}

The most commonly reported complications with IO line placement result from fluid infiltration of the subcutaneous tissue secondary to catheter misplacement. ${ }^{1,3}$ Extravasated fluid may lead to tissue damage, compartment syndrome, and even tissue necrosis in some cases. ${ }^{1,4,5}$ Localized cellulitis and the formation of subcutaneous abscesses also have been reported, albeit rarely. ${ }^{3,5}$

In our retrospective cohort review, we identified an additional potential complication of IO line placement that has not been widely reported-development of large traumatic bullae. It is most likely that this patient's IO catheter became dislodged, resulting in extravasation of fluids into the dermal and subcutaneous tissues.

Our findings support the previously noted complication rate of less than 1\% following IO line placement, with an overall complication rate of $2.7 \%$ that included only 1 patient with a cutaneous complication. ${ }^{2}$ Given this low incidence, providers may not be used to recognizing such complications, leading to delayed or incorrect diagnosis of these entities. While there are certain conditions in which IO insertion is contraindicated, including severe bone diseases (eg, osteogenesis imperfecta, osteomyelitis), overlying cellulitis, and bone fracture, these conditions are rare and can be avoided in most cases by use of an alternative site for needle insertion. ${ }^{2}$ Due to the widespread utility of this tool and its few contraindications, its use in hospitalized patients is rapidly

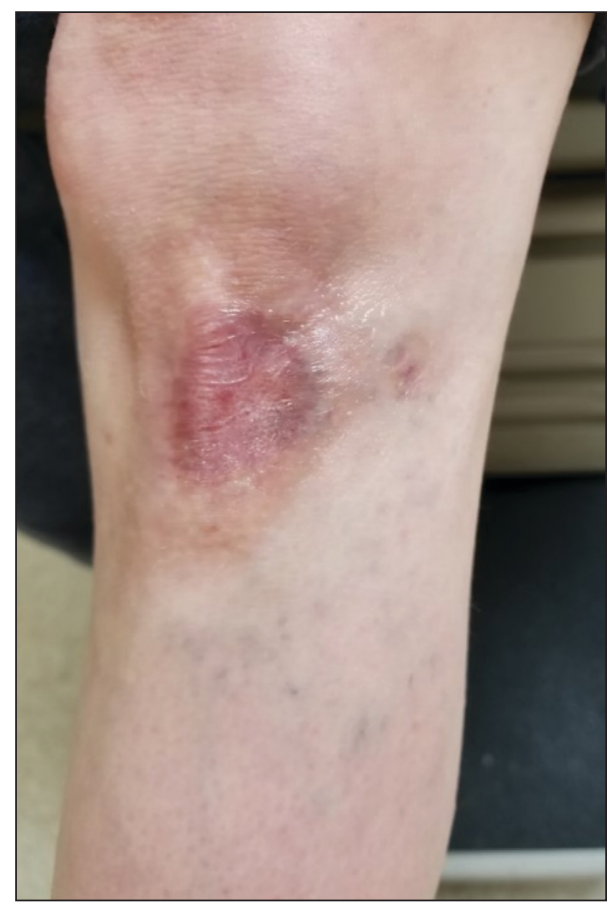

FIGURE 2. Seven months after the bullae were incised and drained, the wound bed appeared to be healing well with surrounding scarring and no residual bleeding or drainage. 
increasing, necessitating a need for quick recognition of potential complications.

From previous data on the incidence of traumatic blisters with underlying bone fractures, there are several identifiable risk factors that could be extended to patients at high risk for developing cutaneous IO complications secondary to the trauma associated with needle insertion, ${ }^{6}$ including wound-healing impairments in patients with fragile lymphatics, peripheral vascular disease, diabetes, or collagen vascular diseases (eg, lupus, rheumatoid arthritis, Sjögren syndrome). Patients with these conditions should be closely monitored for the development of bullae. ${ }^{6}$ While the patient we highlighted in our study did not have a history of such conditions, her history of cardiac disease, recent resuscitation attempts, and immunosuppression certainly could have contributed to suboptimal tissue agility and repair after IO line placement.

\section{Conclusion}

Intraosseous access is a safe, effective, and reliable option for vascular access in both pediatric and adult populations that is widely used in both prehospital (ie, paramedic administered) and hospital settings, including intensive care units, emergency departments, and any acute situation where rapid vascular access is necessary.
This retrospective chart review examining the incidence and types of cutaneous complications associated with IO line placement at a level I trauma center revealed a total complication rate similar to those reported in previous studies and also highlighted a unique postprocedural cutaneous finding of traumatic bullae. Although no unified management recommendations currently exist, providers should consider this complication in the differential for hospitalized patients with large, atypical, asymmetric bullae in the absence of an alternative explanation for such skin findings.

\section{REFERENCES}

1. Day MW. Intraosseous devices for intravascular access in adult trauma patients. Crit Care Nurse. 2011;31:76-90. doi:10.4037/ccn2011615

2. Petitpas F, Guenezan J, Vendeuvre T, et al. Use of intra-osseous access in adults: a systematic review. Crit Care. 2016;20:102. doi:10.1186/s13054-016-1277-6

3. Desforges JF, Fiser DH. Intraosseous infusion. $N$ Engl J Med. 1990;322:1579-1581. doi:10.1056/NEJM199005313222206

4. Simmons CM, Johnson NE, Perkin RM, et al. Intraosseous extravasation complication reports. Ann Emerg Med. 1994;23:363-366. doi:10.1016/S0196-0644(94)70053-2

5. Paxton JH. Intraosseous vascular access: a review. Trauma. 2012;14: 195-232. doi:10.1177/1460408611430175

6. Uebbing CM, Walsh M, Miller JB, et al. Fracture blisters. West J Emerg Med. 2011;12:131-133. doi:10.1016/S0190-9622(09)80152-7 